\title{
A study of the layered microstructure and electrical resistivity of an injection-molded metallic fiber-filled polymer composites
}

\begin{abstract}
Haihong $\mathrm{Wu}^{1}$, Xingzhi Sun ${ }^{1}$, Gangyi $\mathrm{Cai}^{1}$ and Zhenfeng Zhao ${ }^{2}$
Injection molding is an important processing method for conductive fiber-filled polymer composites. The disadvantage of this approach is that the electrical resistivity of the molded part increases with processing because the fiber is easily broken down under the action of flow shear stresses. In order to effectively decrease the electrical resistivity of the molding, we investigated the layered microstructure of a molded part made from stainless steel fiber-filled polypropylene and measured the corresponding resistivity of each layer for different processing parameters. We determined the optimal injection pressure to maintain the aspect ratio of the fibers and their mass fraction in each layer. The packing pressure raised the mass fraction of fibers in each layer, but the pressure also had an obvious effect on the resistivity of the skin layer and little effect on the core layer. The transcrystallinity close to the fibers was found in the subskin layer of the part. A morphological change in transcrystallinity was observed for different processing parameters, and its effect on the resistivity was assessed.
\end{abstract}

Polymer Journal (2012) 44, 1138-1144; doi:10.1038/pj.2012.83; published online 23 May 2012

Keywords: conductive polymer composite; injection molding; resistivity; stainless steel fiber

\section{INTRODUCTION}

Polymers can be obtained with specific electrical characteristics from low to high conductivity by the addition of electrically conductive fillers. Thus, conductive polymers are frequently desired for various applications in which the conductivity of metals is not required. Carbon black-filled polymer composites are one of the most common conductive polymer composites (CPCs) that are often used as antistatic materials. ${ }^{1,2}$ In addition to carbon black, metallic fibers are often applied to improve the electrical conductivity of polymers. ${ }^{3-6}$ The percolation thresholds of conductive fiber-filled polymers decrease with increasing aspect ratio of the filler, an effect which has been confirmed both experimentally and theoretically. ${ }^{7,8}$

The most common technique for forming complex polymer products is injection molding. Many processing parameters have a significant influence on the electrical properties of injection-molded CPC. ${ }^{9-11}$ In light of research findings on the microstructure and electrical properties of CPCs, ${ }^{12-14}$ some strategies to improve electrical properties of CPCs have been developed, such as double-percolated networks induced by immiscible polymer blends, enhancing interparticle connectivity and adding conductive fibers instead of particles. Apart from these approaches, control of the processing parameters is another important way to improve the electrical properties of CPCs. Some research showed that the electrical resistivity of an injection-molded CPCs filled with multiwalled carbon nanotubes could be changed over a wide range (up to 6 orders of magnitude) by varying the injection molding velocity and melt temperature, but the packing pressure was found to have little influence. ${ }^{15}$ However, another study showed that the packing pressure significantly affected the electrical properties on injection-molded stainless steel fiber (SSF)-filled polycarbonate composites. ${ }^{9}$ Different studies drew contradicting conclusions. In contrast to multiwalled carbon nanotube-filled polymer composites, the aspect ratio of fibers is inevitably reduced under the action of flowing shear stresses during injection molding, which always leads to a decrease in the conductivity of the molded part. Among the various processing parameters, it has been further reported that an increase in the processing temperature contributes to an increase in filler agglomeration and a reduction in breakage because of a decrease in flowing shear stresses. ${ }^{16}$ However, the study did not mean whether an increase in processing temperature would lead to an increase in the electrical conductivity for all polymer composites. For some composites, it is possible to preserve the filler aspect ratio such that the effect of agglomeration is minor, which is related to the nature of the filler. It has been confirmed that the conductivity of SSF-filled acrylonitrile-butadiend-styrene was higher than that of nickel-coated carbon fiber-filled acrylonitrile-butadiend-styrene with the same length and mass fraction of fiber, ${ }^{11}$ because SSF twisted easily and connected to form a three-dimensional network, whereas the

${ }^{1}$ School of Mechanical and Electrical Engineering, Henan University of Technology, Zhengzhou, China and ${ }^{2}$ National Engineering Research Center for Advanced Polymer Processing Technology, Zhengzhou University, Zhengzhou, China

Correspondence: Professor H Wu, School of Mechanical and Electrical Engineering, Henan University of Technology, 195 Zhongyuan Road, Zhongyuan District, Zhengzhou, Henan 450007, China.

E-mail: hellen1109@gmail.com or whhholly@yahoo.com.cn

Received 5 December 2011; revised 17 March 2012; accepted 25 March 2012; published online 23 May 2012 
nickel-coated carbon fiber often broke into straight fragments during processing. Taking the aspect of the fiber into account, only the packing pressure would significantly affect the conductivity of the molded part. However, the packing pressure could increase the crystallinity of the matrix, density of the composite and orientation of the fibers. The overall effect of packing pressure on the resistivity of the molded part has not been ascertained.

In our previous study of injection-molded carbon black-filled polypropylene (PP), we found that the injection pressure and packing pressure had different impacts on the resistivity of the skin layer, subskin layer and core layer of the molded part. A high injection pressure did not improve the electrical properties of the part because of a discontinuous band of carbon black particles that formed at the subskin and core layers, and a high packing pressure was beneficial to form a good conductive path in the matrix and improve the electrical properties of the part if the injection pressure was held constant. ${ }^{17}$ However, during our experiments on injection-molded SSF-filled PP, some interesting phenomena were observed. The first was the effect of injection pressure. In contrast to the theoretical speculation, the injection pressure did not improve the electrical properties of the molded part, because the pressure was below a critical value. The second phenomenon was the effect of packing pressure. As the molded part always presented a layered microstructure, the packing pressure had different impacts on the layers. The third observation was the effect of crystallization of the matrix under varying processing parameters.

To explore the relationships among the processing parameters, microstructure and electrical performance of the molded part, we tested a series of injection molding conditions, then observed the microstructure at different positions on the part, such as the skin layer, subskin layer and core layer, and measured each corresponding electrical property. The purpose of the study was to determine the influence of the processing conditions on the electrical conductivity and the microstructure of the molded part and to ascertain the processing parameters that optimized the electrical properties of the part for commercial applications.

\section{EXPERIMENTAL PROCEDURE}

\section{Materials}

For the experiments, 304 SSFs were used (Huitong Advanced Material Company, Hunan Province, Changsha, China). The fiber diameter was $11 \mu \mathrm{m}$, and its resistance was $6.07 \mathrm{~K} \Omega \mathrm{m}^{-1}$. PP was obtained in pelletized form from the Sinopec Group (Nanjing, China). The melting point of the PP used was $165^{\circ} \mathrm{C}$. Its melt flow index was $12 \mathrm{~g}$ per $10 \mathrm{~min}$, and the density was $0.92 \mathrm{~g} \mathrm{~cm}^{-3}$.

\section{Fabrication of composite pellets}

Pellets were made by using an SHJ35 twin-screw plastic pelleter with which the fibers were coated by PP with a cladding mold as shown in Figure 1. The speed of the screw was 30 r.p.m., and the temperature of the coolant was $30^{\circ} \mathrm{C}$. The temperatures of the feeding section, fluxing section and homogenizing section of the barrel were 160,180 and $200^{\circ} \mathrm{C}$, respectively. The temperature of the mold was $200^{\circ} \mathrm{C}$. Before extrusion, SSF was chemically modified to form an active agent on its surface and improve the wettability with PP. The volume fraction of SSFs was $60 \%$. After extrusion, the composite was cut into pellets 3-mm long.

\section{Injection molding}

Standard, plastic tensile samples were injected with the composite pellets mixed with PP. The mass fraction of SSF in the composite was $10 \%$. The injection pressure was changed from 30 to $70 \mathrm{MPa}$ and the packing pressure decreased from 0 to $30 \mathrm{MPa}$. The temperature of the melt was $200{ }^{\circ} \mathrm{C}$ and that of the mold was $45^{\circ} \mathrm{C}$.

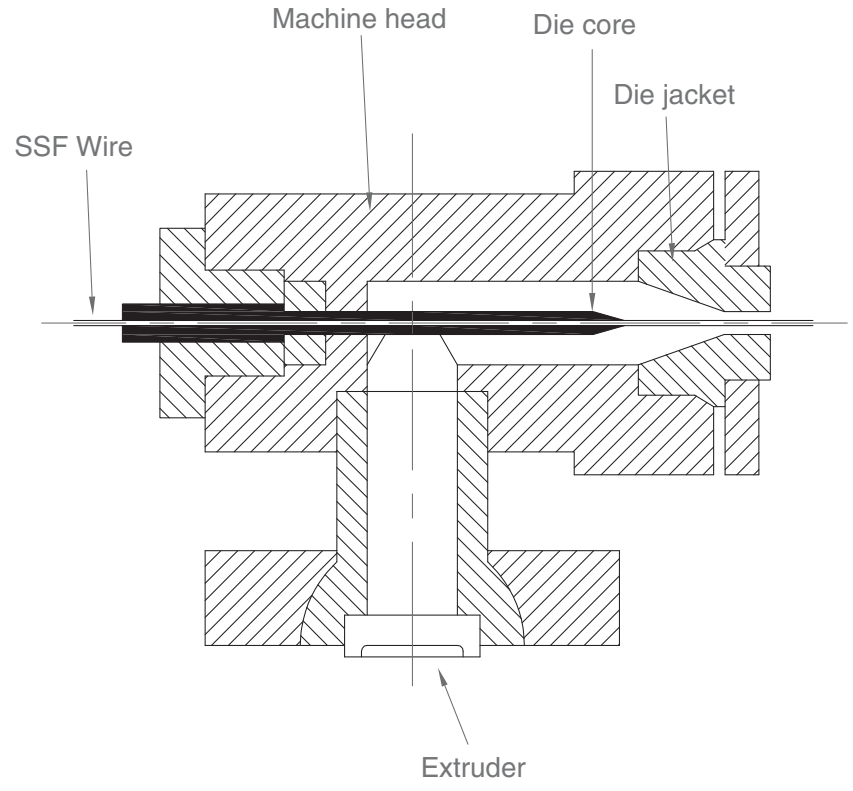

Figure 1 The schematic map of cladding mold. The figure describes the device that is used to fabricate pellets of SSF-filled PP composites. A full color version of this figure is available at Polymer Journal online.

\section{Microstructure analysis}

Rectangular samples, cut from the standard plastic tensile molded part, were milled with a numerically controlled milling machine from the top of the part to observe its microstructure at different thicknesses. The thickness of the first layer removed was $0.1 \mathrm{~mm}$. The exposed surface after milling was named the skin layer surface of the molded part. Next, a layer 0.3 -mm thick was removed and the exposed surface was named the subskin layer. The core layer surface was obtained after removing a final layer of $1.3 \mathrm{~mm}$ in thickness from the molded part. The microstructure of each layer was examined using a Quanta FEG 250 Scanning Electron Microscope (FEI, Hillsboro, OR, USA). The mass fraction of SSFs in each layer was calculated to investigate the effect of processing conditions on the distribution of fibers in the matrix. The density of each sample was measured using an electronic balance.

\section{Measurement of resistivity}

The volume resistivity of each layer was measured in the thickness direction at room temperature using a standard, two-terminal DC resistor. The sample size was $10 \times 10 \times 0.2 \mathrm{~mm}^{3}$ for the skin layer, $10 \times 10 \times 0.4 \mathrm{~mm}^{3}$ for the subskin layer and $10 \times 10 \times 0.8 \mathrm{~mm}^{3}$ for the core layer. Then, the sample was affixed to a slide using polyimide tape. Silver paste was used to ensure good contact between the surface of the sample and copper electrodes. The resistivities of three samples for each layer were measured, and the reported resistivity was a mean value of the three measurements. To obtain an accurate measure of resistivity, the sample was dried in a vacuum furnace at $100{ }^{\circ} \mathrm{C}$ for $2 \mathrm{~h}$ to eliminate the effect of moisture on the measured resistivity.

\section{Measurement of crystallinity}

The crystallinity of the PP matrix in each layer was determined by differential scanning calorimeter (DSC) using an NETZSCH5 DSC200F3 instrument (NETZSCH-Gerätebau GmbH, Selb, Germany). The heating rate was $5 \mathrm{~K}$ per min. Sample mass was $7 \mathrm{mg}$ and that crucible was $\mathrm{Al}$ with a pierced lid.

\section{RESULTS AND DISCUSSION}

For injection-molded SSF-filled PP, the experimental results showed that the volume resistivity mainly depended on three factors, which include the aspect ratio, mass fraction and connectivity of the fiber in the matrix. 

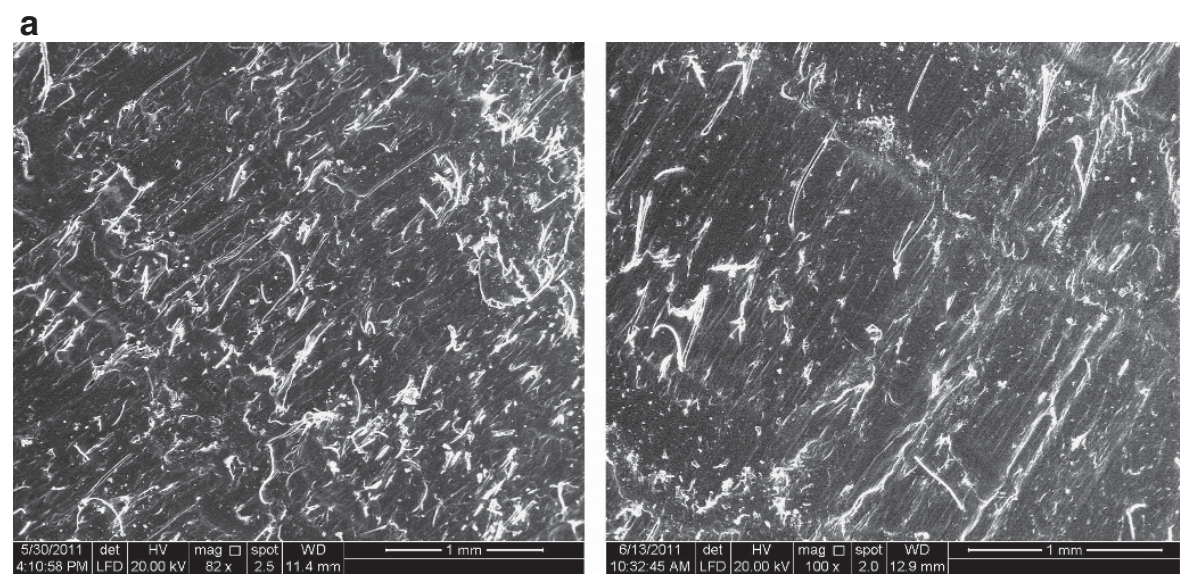

Skin layer

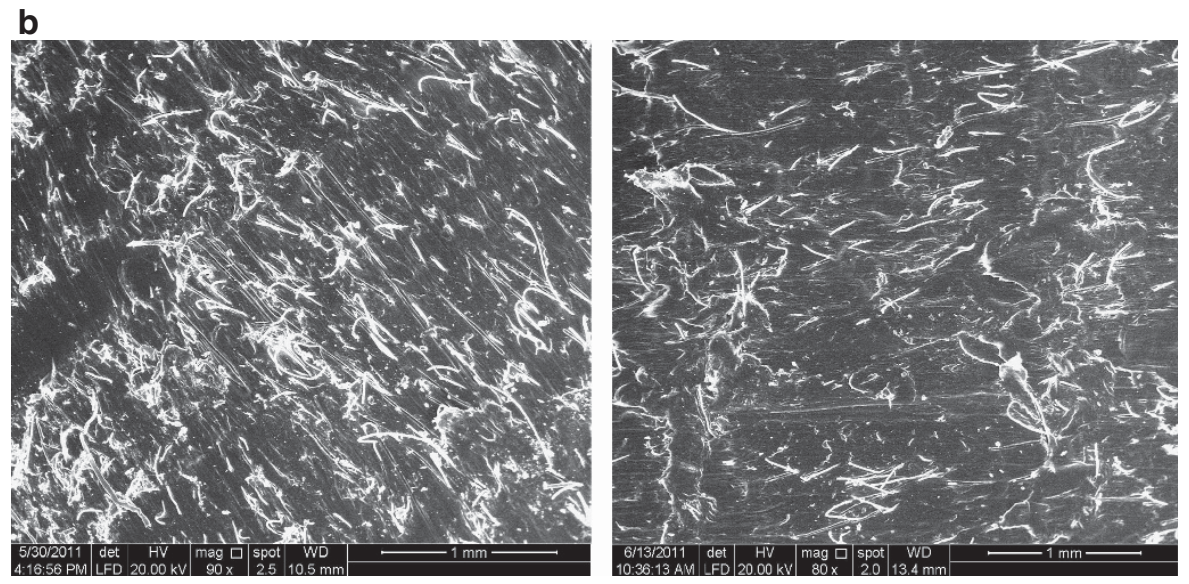

Sub-skin layer
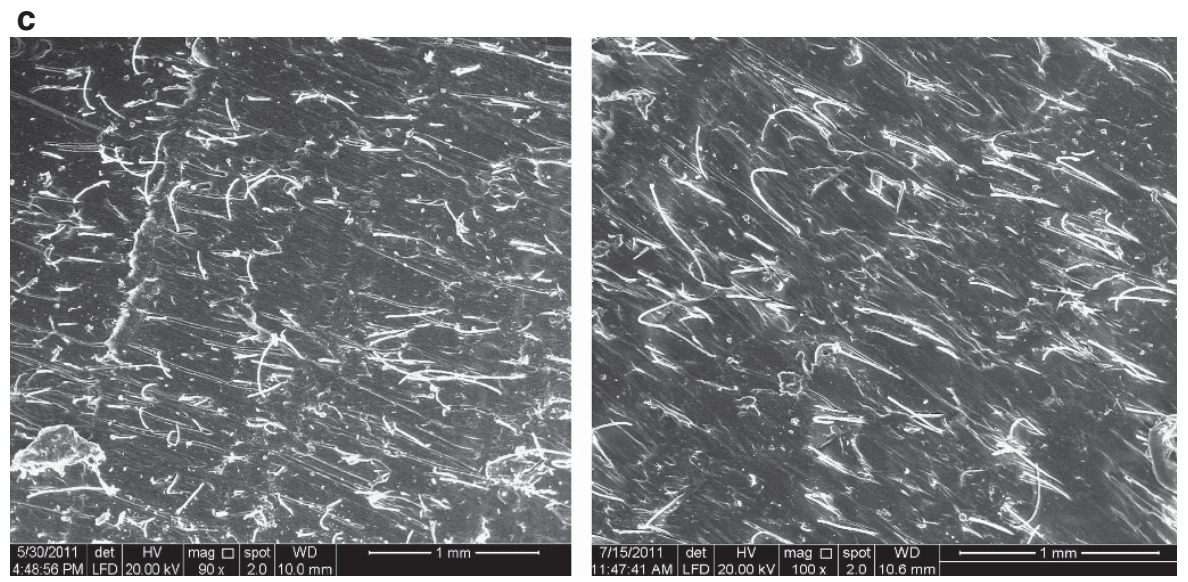

Core layer

Figure 2 The fiber distribution in the matrix at different layers. (Note that: injection pressure is $40 \mathrm{MPa}$ for the left and $30 \mathrm{MPa}$ for the right.) The figure illustrates the microstructures of the skin layer (a), subskin layer (b) and core layer (c) of the molded part, which is helpful to understand the characteristics of the layered microstructure of the part and the changes in the microstructure caused by the processing parameters.

\section{The influence of injection pressure}

As the flow shear stress of the melt is different along the thickness direction of the molded part, the fiber distribution and its aspect ratio were different in each layer. Scanning electron micrographs of samples prepared under different injection pressures without packing pressure are shown in Figure 2.
Based on the mechanics of composite materials, the mass fraction of SSFs, $w_{\mathrm{f}}$, in each layer in terms of the density of the composites can be expressed as

$$
w_{\mathrm{f}}=\frac{\left(\rho_{\mathrm{m}}-\rho_{\mathrm{c}}\right) \rho_{\mathrm{f}}}{\left(\rho_{\mathrm{m}}-\rho_{\mathrm{f}}\right) \rho_{\mathrm{c}}}
$$


Table 1 The mass fraction of SSF at each layer

\begin{tabular}{llllr}
\hline & & \multicolumn{3}{c}{$\begin{array}{c}\text { The mass fraction of SSF } \\
\text { in different layers (\%) }\end{array}$} \\
\cline { 3 - 5 } $\begin{array}{l}\text { Injection } \\
\text { pressure (MPa) }\end{array}$ & $\begin{array}{c}\text { Packing } \\
\text { pressure (MPa) }\end{array}$ & Skin layer & Subskin layer & Core layer \\
\hline 30 & 0 & 9.21 & 10.15 & 9.91 \\
40 & 20 & 9.27 & 10.83 & 10.01 \\
& 0 & 9.3 & 10.19 & 9.96 \\
50 & 20 & 9.68 & 11.2 & 10.08 \\
& 0 & 9.38 & 10.36 & 10.15 \\
& 20 & 9.66 & 11.51 & 10.66 \\
\hline
\end{tabular}

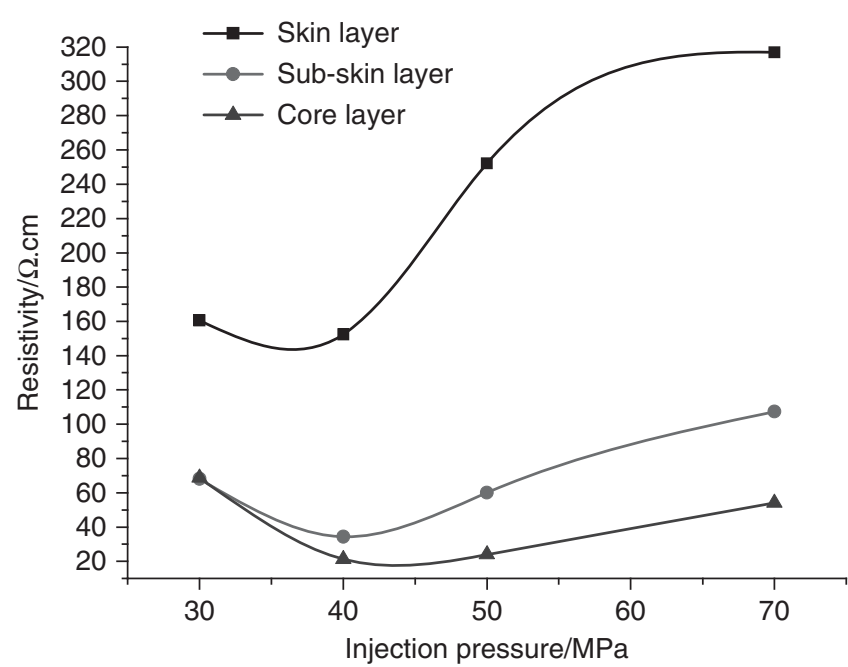

Figure 3 Effect of injection pressure on the resistivity of each layer of the molding. The figure illustrates the effect of injection pressure on the resistivity of each layer of the molded part. A full color version of this figure is available at Polymer Journal online.

where $\rho_{\mathrm{c}}, \rho_{\mathrm{f}}$ and $\rho_{\mathrm{m}}$ are the densities of the composite, fiber and matrix, respectively. The results calculated were shown in Table 1 .

Under the processing conditions without packing pressure, the results in Table 1 reveal that the mass fraction of SSFs in the matrix could be raised for all layers with an increase in injection pressure. For different layers of a molded part, the mass fraction of SSFs was lowest at skin layer and highest in the subskin layer. However, contrary to the results of the SSF mass fraction at each layer, the core layer presented the lowest resistivity, as shown in Figure 3.

There were three reasons for these results. The first reason was that the flow shear stress in the core layer was smaller than in both the skin layer and subskin layer during filling of the mold cavity. Therefore, the aspect ratio of SSFs at the core layer, as shown in Figure 2, was greater than that in other layers during processing. The second explanation was that the SSFs bend easily, thereby reducing the degree of orientation of the fibers because SSFs have good flexibility. It was known that orientation of the conductive filler is not beneficial to the conductivity of CPCs. This is why injection-molded CPCs are often fabricated with SSF-filled polymer rather than carbon fiber-filled polymer. The third reason might be related to the crystallinity of the matrix. Conductive filler has two preferred locations during processing of the CPCs. One is the boundary between the amorphous zone
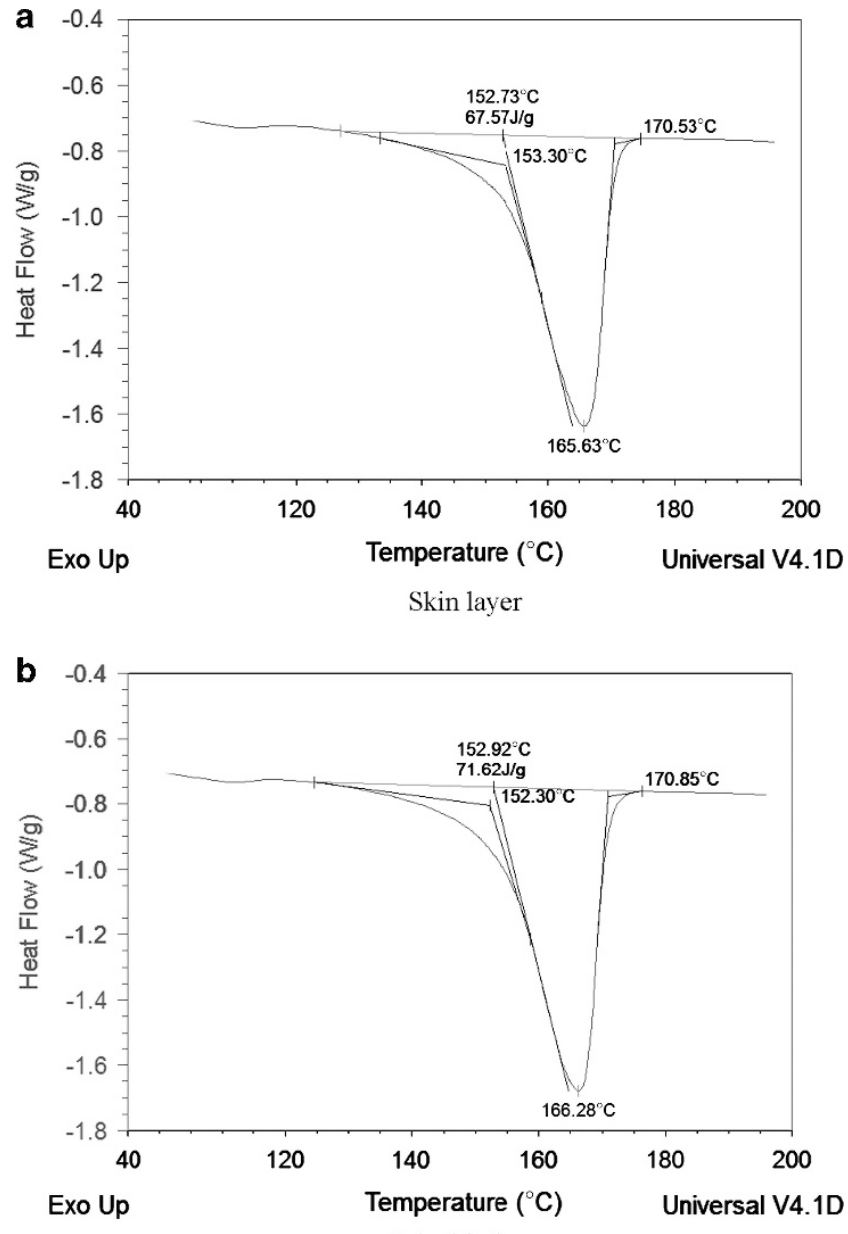

Sub-skin layer

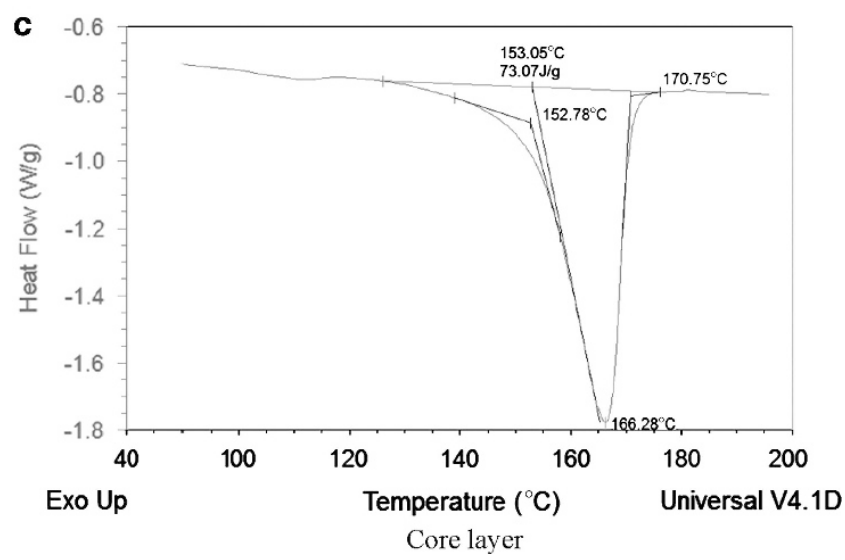

Figure 4 The crystallinity of the matrix at different layers. (Note: injection pressure is $40 \mathrm{MPa}$ and packing pressure $0 \mathrm{MPa}$ ). The figure illustrates the results of DSC that were used to measure the crystallinity of the matrix in different layers, that is, skin layer (a), subskin layer (b) and core layer (c). The results in Figure 4 were obtained at a $40 \mathrm{MPa}$ injection pressure and without packing pressure. A full color version of this figure is available at Polymer Journal online.

and the crystalline zone, and the other is the amorphous zone of the polymer. No matter where the fibers are located, a higher crystallinity of the matrix would be helpful to improve the conductivity of CPCs. The results of DSC for each layer, as shown in Figure 4, support this conclusion. 
The crystallinity of the matrix in different layers was obtained by the following equation

$$
\theta=H_{\mathrm{c}} / H_{0} \times 100 \%
$$

where $\theta$ denotes the crystallinity, $H_{\mathrm{c}}$ the heat absorbed by melting of the crystalline phase and $H_{0}$ the heat under the condition of full crystallization of PP. $H_{0}$ is $202 \mathrm{Jg}^{-1}$. The calculated results showed that $\theta_{\text {skin }}<\theta_{\text {subskin }}<\theta_{\text {core }}$

The above results show that lowering the injection pressure below a critical value could not further decrease the resistivity of the molded part, although the aspect ratio of the SSFs was large. Thus, it was important to optimize the processing parameters in order to maintain the aspect ratio of the SSFs and the connectivity between the fibers, which could be ensured by the mass fraction and distribution of fibers in the matrix. Based on the relationship between the crystallinity of the matrix and the resistivity of the CPC, some methods that could increase the crysallinity of the matrix might decrease the resistivity of the molded part, such as raising the temperature of the mold wall.

\section{The influence of packing pressure}

Under the action of packing pressure, the situation seems more complex for each layer. The results in Table 1 illustrate that the mass fraction of SSFs in the matrix in each layer of the molded part increased with increasing packing pressure, if the injection pressure was held constant. But the resistivity in the skin layer did not decrease under the higher packing pressure, as shown in Figure 5. On the contrary, the resistivity of the skin layer increased rapidly with increasing packing pressure. This effect was mainly related to the orientation of SSFs in the direction of flow, as shown in Figure 6. The strong orientation of the fibers induced by the packing pressure damaged the conductive network.

Figure 5 shows that the packing pressure had a relatively small effect on the resistivity of the subskin layer and, especially, the core layer. We believe that the lower resistivity of the subskin layer is determined by the higher mass fraction of SSFs and that of the core layer is mainly related to the higher crystallinity of the matrix that is induced by packing pressure. The results of DSC experiments, as shown in Figure 7, confirmed this supposition.

Our experimental results show that the packing pressure did not have a significant effect on the resistivity of the subskin layer or the core layer of the molded part. In practice, the part might have good electrical properties after removal of the thin skin layer. Therefore, the packing pressure could be optimized for other purposes, such as density of the CPC, the mechanical properties of the part or warpage of the part. In this respect, the optimized processing parameters would be a lower injection pressure matched with a moderate packing pressure.

\section{The influence of transcrystallinity}

During our experiments, a transcrystalline phase-that is, a crosshatched structure composed of lamellae parallel and perpendicular to the fiber surface-was observed close to the fibers. The results are shown in Figure 8.

Figure 8 shows that transcrystallinity formed in the matrix close to the fibers because of interaction between the fibers and the matrix. The results shown in Figure 8 reveal that transcrystallinity would be found with an increase in the injection pressure (Figure $8 \mathrm{~b}$ ) or an increase in the packing pressure (Figure 8c). These results were supported by other researchers who strongly suggested that the shear stress could induce transcrystallinity. ${ }^{18,19}$ Compared with the crystallinity of the matrix, the transcrystallinity had a higher degree

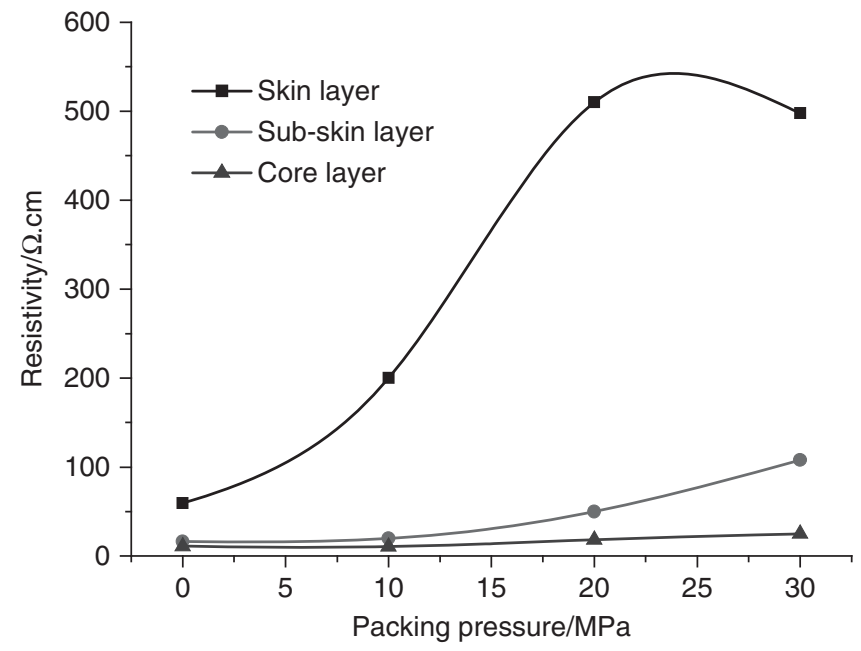

Figure 5 Effect of packing pressure on the resistivity at each layer of the molding (injection pressure: $40 \mathrm{MPa}$ ). The figure describes the effect of the packing pressure on the resistivity of each layer of the molded part. It shows that packing pressure has great influence on the resistivity at the skin layer but little effect on the core layer. A full color version of this figure is available at Polymer Journal online.

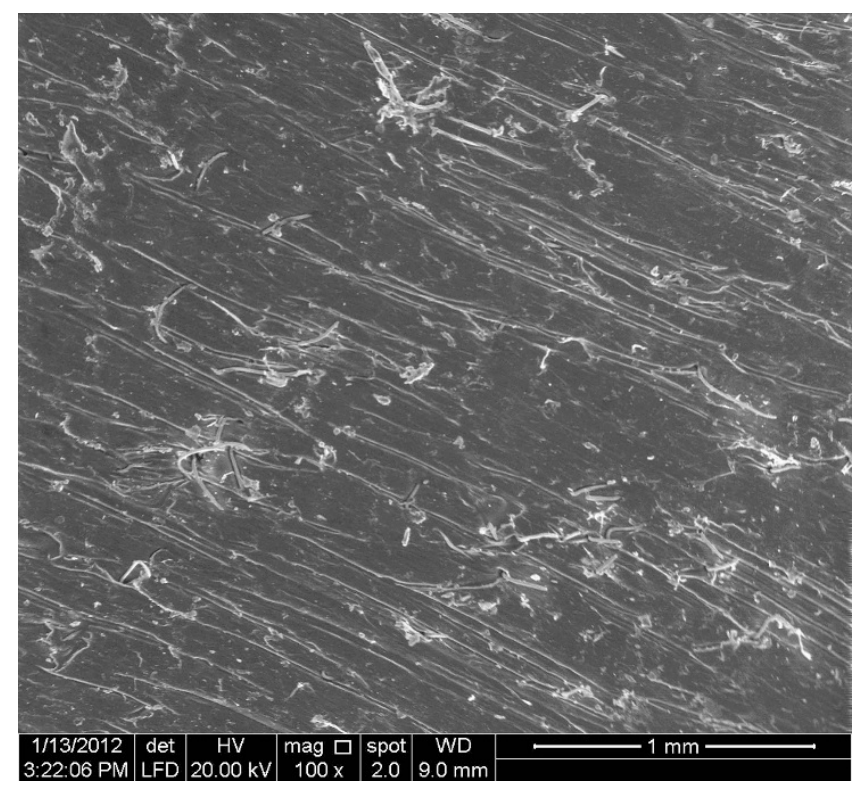

Figure 6 Packing-induced orientation the SSF at the skin layer (injection pressure is $30 \mathrm{MPa}$ and packing pressure $20 \mathrm{MPa}$.). The figure illustrates the orientation of the SSFs in the skin layer induced by the packing pressure. The strong orientation of the fibers increases the resistivity of the skin layer.

of order, which resulted from very compact crystal packing and possibly from a preferred crystalline alignment. The compacted crystal could impede the transport of charge carriers in the CPC. Therefore, the electrical response was sensitive to the presence of transcrystallinity in the CPC. Based on the assumptions for composite materials, the electrical properties are determined by the proportional contributions of the fiber, crystalline matrix and transcrystallinity. The resistivity of the composites increase with an increase in transcrystallinity. This conclusion might explain the fact that the resistivity of each layer of the molded part did not decrease with an increase in the packing pressure, although the mass fraction 
a

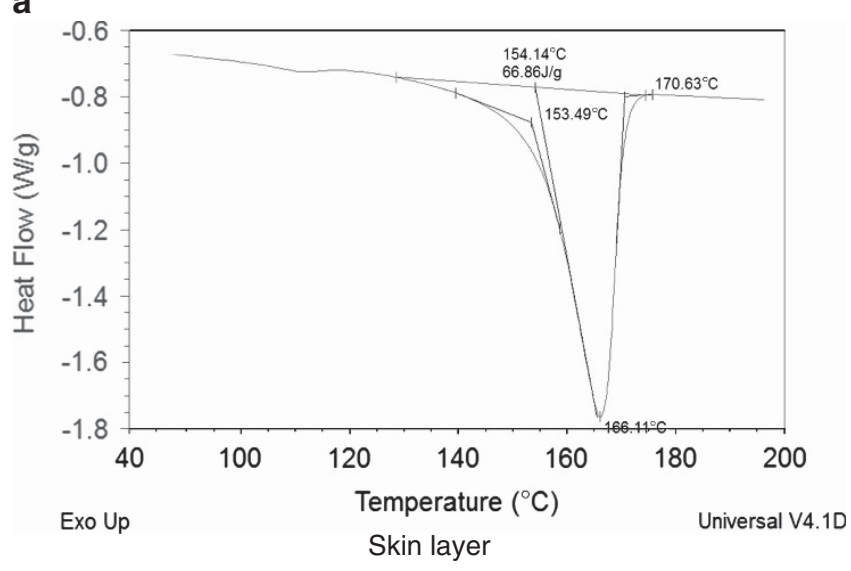

b

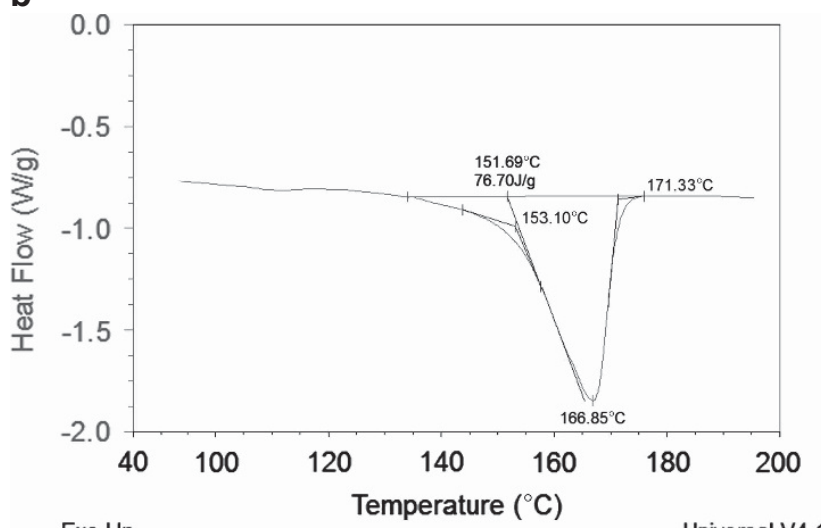

Sub-skin layer

C

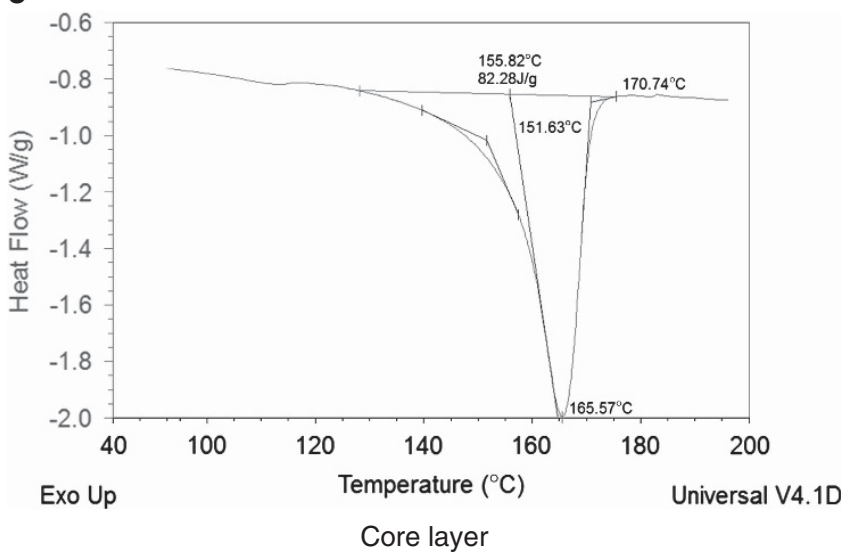

Figure 7 The crystallinity of the matrix at different layers. (Injection pressure is $40 \mathrm{MPa}$ and packing pressure $20 \mathrm{MPa}$ ). The figure illustrates the results of DSC obtained under the higher packing pressure that were used to elucidate the effect of the packing pressure on the resistivity of each layer, that is, skin layer (a), subskin layer (b) and core layer (c). A full color version of this figure is available at Polymer Journal online.

of fibers in each layer was larger than that of samples processed without packing pressure. Likewise, this reasoning might explain why the subskin layer did not have the lowest resistivity, even though it had the highest fiber mass fraction, because the transcrystalline phase often formed in this layer. In our experiments, transcrystallinity was

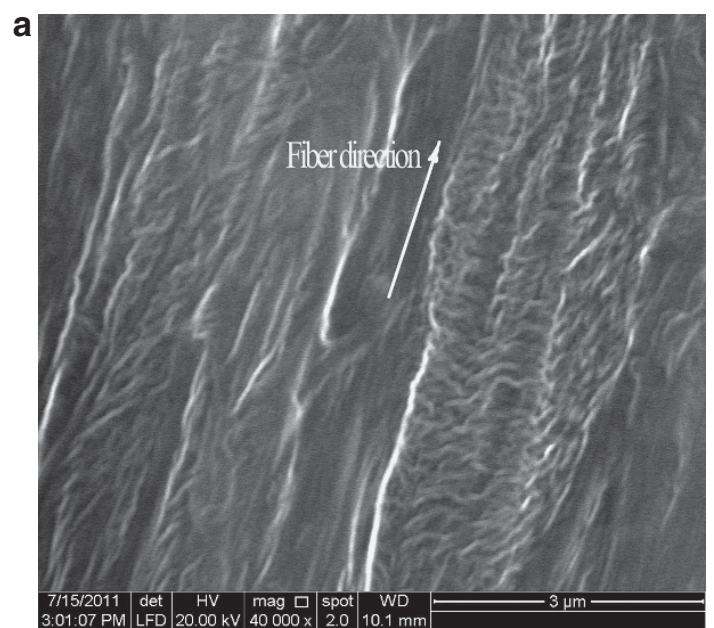

Injection pressure is $40 \mathrm{MPa}$ without packing

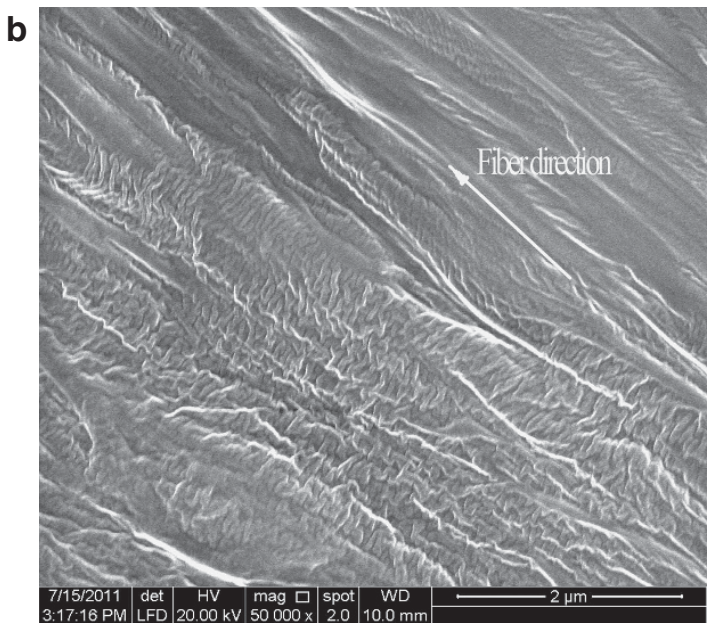

Injection pressure is $50 \mathrm{MPa}$ without packing

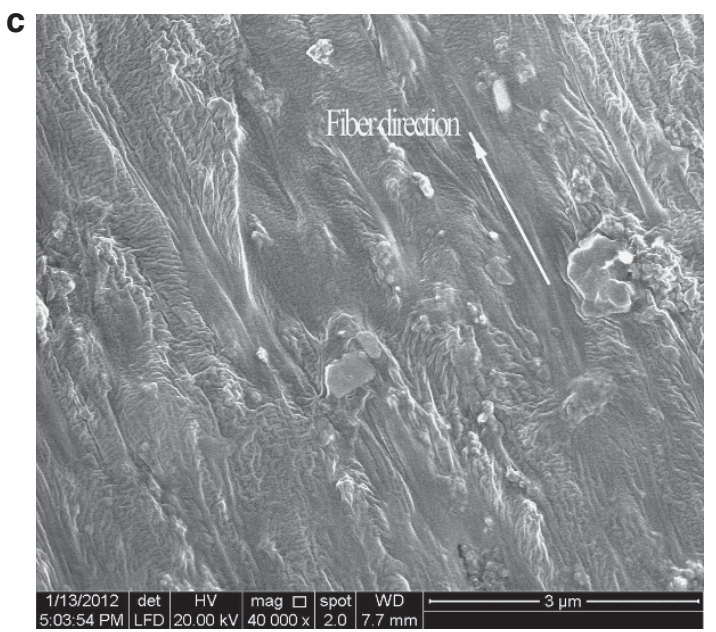

Injection pressure is $40 \mathrm{MPa}$ with $20 \mathrm{MPa}$ packing pressure

Figure 8 Transcrystallinity formed at subskin layer close to the fiber. The figure illustrates the transcrystalline phase that formed in the subskin layer close to the fibers and morphological changes in that phase with varying processing parameters. (a) Crosshatched structure composed of lamellae parallel and perpendicular to the fiber surface. (b) Crosshatched structure was compacted with the increase of injection pressure. (c) Crosshatched structure was compacted with the increase of packing pressure. 
occasionally observed in the skin layer but was seldom found in the core layer. How the transcrystallinity affects the resistivity of the injection-molded SSF-filled polymer composite should be investigated further.

The mechanism of formation of transcrystallinity was the subject of speculation based on the theory put forth by Hobbs ${ }^{20}$ and Thomason and Van Rooyen. ${ }^{21}$ Additional shear stress, which was induced by the unmatched thermal expansion coefficient between the matrix and the fiber, leads to transcrystallinity at the interface of the matrix and the fibers. Perhaps, the steric effect of the fibers on the matrix is another reason for the formation of transcrystallinity. ${ }^{22}$

\section{CONCLUSIONS}

The results of this study showed that the resistivity of injectionmolded SSF-filled PP was not uniform in the molded part. In the skin layer of the part, the resistivity was higher than in other layers, whereas in the core layer, the resistivity was at a minimum. The injection pressure had an important influence on the resistivity of each layer. The resistivity of the each layer of the molded part increased with an increase in the injection pressure. However, the lower injection pressure did not always lead to a decrease in the resistivity of the molded part because of the poor connectivity of SSFs. There was an optimum injection pressure that lead to a balance between the aspect ratio of the fibers and the connectivity between the fibers in the matrix. The packing pressure had a different, complex effect on the resistivity of each layer. For the skin layer, because packing pressure induced strong orientation of the fibers, the resistivity of this layer obviously increased with higher packing pressure, although the mass fraction of fibers in the matrix was high. However, the packing pressure had a relatively small effect on the resistivities of the subskin layer and the core layer of the molded part, which are related to the higher mass fraction of SSFs and the higher crystallinity induced by the packing pressure. In the subskin layer, transcrystallinity was always present and refined with an increase of either the injection pressure or the packing pressure. The resistivity of the composites could be increased with an increase in the transcrystallinity. This conclusion can be used to describe the influence of the processing conditions on the resistivity of each layer of the molded part.

\section{CONFLICT OF INTEREST}

The authors declare no conflicts of interest.

\section{ACKNOWLEDGEMENTS}

We appreciate the National Natural Science Foundation of China for financial support (number: 51075120). We also appreciate the financial support of the Zhengzhou Scientific and Technological Innovation Project (number: 10CXTD519).
1 Narkis, M., Lidor, G., Vaxman, A. \& Zuri, L. New injection moldable electrostatic dissipative (ESD) composites based on very low carbon black loadings. J. Electrostat 47, 201-214 (1999).

2 Jiang, X., Bin, Y., Kikyotani, N. \& Matsuo, M. Thermal electrical and mechanical properties of ultra-high molecular weight polypropylene and carbon filler composites. Pol. J. 38, 419-431 (2006).

3 Bigg, D. M. Mechanical and conductive properties of metal fibre-filled polymer composites. Polym. Eng. Sci. 19, 1188-1192 (1979).

4 Bridge, B., Folkes, M. J. \& Jahankhani, H. Low voltage electrical properties of polypropylene filled with stainless steel fibres and a model of sample conductivity based on fibre geometry. J. Mater. Sci. 24, 1479-1485 (1989).

5 Bridge, B., Folkes, M. J. \& Jahankhan, H. I. Electrical conduction phenomena between adjacent stainless steel fibres in a thermoplastic matrix. J. Mater. Sci. 23, 1955-1960 (1988)

6 Li, Y.-J., Xu, M., Feng, J.-Q. \& Dang, Z. Dielectric behavior of a metal-polymer composite with low percolation Threshold. Appl. Phys. Lett. 89, 072902 (2006).

7 Sun, J.-S., Gokturk, H. S. \& Kalyon, D. M. Volumer and surface resistivity of low density polyethylene filled with stainless steel fibers. J. Mater. Sci. 28, 364-366 (1993).

8 Tan, S. T., Ming, M. Q., Rong, M. Z., Zeng, H. M. \& Zhao, F. M. Properties of metal fibre filled thermoplastics as candidates for electromagnetic interference shielding. Polym. Polym. Compos. 9, 257-262 (2001).

9 Chen, C.-S., Chen, W.-R., Chen, S.-C. \& Chien, R.-D. Optimum injection molding processing condition on EMI shielding effectiveness of stainless steel fiber filled polycarbonate composite. Int. Comm. Heat Mass Transfer 35, 744-749 (2008).

10 Yang, S. Y., Chen, C. Y. \& Parng, S. H. Effects of conductive fibers and processing conditions on the electromagnetic shielding effectiveness of injection molded composites. Polym. Compos. 23, 1003-1013 (2002).

11 Chen, S. C., Lee, P. H., Huang, J. S. \& Chien, R. D. Effects of molding conditions on the electromagnetic interference performance of conductive ABS parts. J. Appl. Polym Sci. 98, 1072-1080 (2005).

12 Thongruang, W., Spontak, R. J. \& Balik, C. M. Bridged double percolation in conductive polymer composites: an electrical conductivity, morphology and mechanical property study. Polymer 43, 3717-3752 (2002).

13 Markov, A., Fiedler, B. \& Schulte, K. Electrical conductivity of carbon black/fibers filled glass-fiber-reinforced thermoplastic composites. Compos. Part A 37, 1390-1395 (2006)

14 Saleem, A., Frormann, L. \& Iqbal, A. Mechanical, thermal and electrical resisitivity properties of thermoplastic composites filled with carbon fibers and carbon particles. J. Polym. Res. 14, 121-127 (2007).

15 Villmow, T., Pegel, S., Pötschke, P. \& Wagenknecht, U. Influence of injection molding parameters on the electrical resistivity of polycarbonate filled with multi-walled carbon nanotubes. Compos. Sci. Technol. 68, 777-789 (2008).

16 Al-Saleh, MH \& Sundararaj, U Processing-microstructure-property relationship in conductive polymer nanocomposites. Polymer 51, 2740-2747 (2010).

$17 \mathrm{Wu}, \mathrm{H}$. , Fen, L., Jiang, A. \& Zhang, B. Effect of the processing of injection molded, carbon black-filled polymer composites on resistivity. Pol. J. 43, 930-936 (2011)

18 Misra, A., Deopura, B. L., Xavier, S. F., Hartley, F. D. \& Peters, R. H. Transcrystallinity in injection molded polypropylene glass-fiber composites. Angew Markomol. Chem. 13, 113-120 (1983).

19 Devaux, E. \& Chabert, B. Nature and origin of the transcrystalline interphase of polypropylene glass-fiber composites after a shearstress. Polym. Commun. 32 464-468 (1991)

20 Hobbs, S. H. Row nucleation of isotactic polypropylene on graphite fibres. Nat. Phys Sci. 234, 12 (1971).

21 Thomason, J. L. \& Vanrooyen, A. A. Transcrystallized interphase in thermoplastic composites Part II Influence of interfacial stress, cooling rate, fibre properties and polymer molecular weight. J. Mater. Sci. 27, 897-907 (1992).

22 Huang, Y. \& Peteramann, J. Transcrystalline growth of thermoplastics and LCPs during isothermal crystallization. J. Appl. Polym. Sci. 55, 981-987 (1995). 